\title{
Thorough characterization of a EUV mask
}

\author{
Hiroyuki Mizuno*a ${ }^{\mathrm{a}}$, Gregory McIntyre ${ }^{\mathrm{b}}$, Chiew-seng Koay ${ }^{\mathrm{b}}$, Martin Burkhardt ${ }^{\mathrm{c}}$, \\ Long $\mathrm{He}^{\mathrm{d}}$, John Hartley ${ }^{\mathrm{e}}$, Corbet Johnson ${ }^{\mathrm{e}}$, Sudhar Raghunathan ${ }^{\mathrm{e}}$, \\ Kenneth Goldberg ${ }^{f}$, Iacopo Mochi ${ }^{\mathrm{f}}$, Bruno La Fontaine ${ }^{\mathrm{g}}$, and Obert Wood ${ }^{\mathrm{h}}$ \\ ${ }^{a}$ Toshiba America Electronic Components, 257 Fuller Road, Albany, NY 12203, USA \\ ${ }^{b}$ IBM Advanced Lithography Research, 257 Fuller Road, Albany, NY 12203, USA \\ IBM Corporation, 2070 Route 52, Hopewell Junction, NY 12533, USA \\ ${ }^{\mathrm{d} S E M A T E C H,} 257$ Fuller Road, Albany, NY 12203, USA \\ ${ }^{\mathrm{e}}$ College of Nanoscale Science and Engineering, 257 Fuller Road, Albany, NY 12203, USA \\ ${ }^{f}$ Lawrence Berkeley National Laboratory, One Cyclotron Road, Berkeley, CA, 94720, USA \\ ${ }^{\mathrm{g}}$ GLOBALFOUNDARIES, 1050 E. Arques Avenue, Sunnyvale, CA 94088, USA \\ ${ }^{\mathrm{h}}$ GLOBALFOUNDARIES, 257 Fuller Road, Albany, NY 12203, USA \\ *contact: hiroyuki.mizuno@,taec.toshiba.com tel: (518) 292-7231
}

\begin{abstract}
We reported that we were successful in our 45nm technology node device demonstration in February 2008 and 22nm node technology node device patterning in February 2009 using ASML's Alpha Demo Tool (ADT) ${ }^{1,2,3}$ In order to insert extreme ultraviolet (EUV) lithography at the $15 \mathrm{~nm}$ technology node and beyond, we have thoroughly characterized one EUV mask, a so-called NOVACD mask.

In this paper, we report on three topics. The first topic is an analysis of line edge roughness (LER) using a mask Scanning Electron Microscope (SEM), an Atomic Force Microscope (AFM) and the Actinic Inspection Tool (AIT) to compare resist images printed with the ASML ADT. The results of the analysis show a good correlation between the mask AFM and the mask SEM measurements. However, the resist printing results for the isolated space patterns are slightly different. The cause of this discrepancy may be resist blur, image log slope and SEM image quality and so on.

The second topic is an analysis of mask topography using an AFM and relative reflectivity of mirror and absorber surface using the AIT. The AFM data show 6 and 7 angstrom rms roughness for mirror and absorber, respectively. The reflectivity measurements show that the mirror reflects EUV light about 20 times higher than absorber.

The last topic is an analysis of a $32 \mathrm{~nm}$ technology node SRAM cell which includes a comparison of mask SEM image, AIT image, resist image and simulation results. The ADT images of the SRAM pattern were of high quality even though the mask patters were not corrected for OPC or any EUV-specific effects. Image simulation results were in good agreement with the printing results.
\end{abstract}

Keywords: EUV, LER, SEM, AFM, AIT (actinic inspection tool), reflectance, SRAM

\section{INTRODUCTION}

EUV lithography is one of the most promising methods for next-generation lithography at the $15 \mathrm{~nm}$ technology node and beyond. But, critical issues such as powerful clean EUV sources, resist line edge roughness (LER), defect-free masks, and mask optical proximity correction (OPC) still require additional development.

OPC for EUV lithography is quite different from OPC for excimer laser lithography, because the data on a EUV mask must be corrected not only for conventional OPC, but also for flare and for mask shadowing. ${ }^{4,5,6,7,8}$ Correcting for mask shadowing, which gives both a critical dimension (CD) error and an edge placement error and is caused by a combination of non-telecentric EUV tool optics and mask topography, is especially important. Because of this it is very important to thoroughly characterize an EUV mask. 
In this paper, we have studied one EUV mask in great detail, a so-called NOVACD mask which was developed for ASML Alpha Demo Tool characterization and for OPC modeling of 22-nm and beyond node logic devices. We show data from the NOVACD mask obtained with a scanning electron microscope (SEM), an atomic force microscope (AFM), and an actinic inspection tool (AIT) and will discuss the following topics:

LER analysis: We compare the LER of a 200-nm line/space feature on the NOVACD mask to the LER of the $4 x$ reduction printed line/space image using an SEM.

Surface analysis: We show AFM images of mirror and absorber areas on the mask for comparison with AIT images. From the AIT images, we have calculated the reflectance ratio of an absorbing area and a reflecting area of the NOVACD mask.

32nm technology node SRAM analysis: We show mask SEM images, AIT images, data from a TEMPEST simulation, and resist images printed with the ADT for comparison with each other.

\section{LER ANALYSIS BY MASK SEM, MASK AFM AND RESIST SEM IMAGE}

\section{$2.1200 \mathrm{~nm}$ line and space evaluation patterns}

The LER analysis of the NOVACD mask was carried out at the center of a $400 \mu \mathrm{m}$ square area which was filled with $200 \mathrm{~nm}$ lines or spaces at various densities $(1,10,20,30,40,50,60,70,80,90$ and $99 \%)$. For example, the $1 \%$ pattern density includes a $200 \mathrm{~nm}$ space and a $19800 \mathrm{~nm}(200 \times 99)$ line; $20 \%$ pattern density includes a $200 \mathrm{~nm}$ space and a $800 \mathrm{~nm}(200 \mathrm{x}$ 4) line; $50 \%$ pattern density includes a $200 \mathrm{~nm}$ space and a $200 \mathrm{~nm}$ line; and $99 \%$ pattern density includes a $200 \mathrm{~nm}$ line and a $19800 \mathrm{~nm}$ space.

\subsection{Mask SEM evaluation and mask AFM evaluation}

The mask AFM measurement was carried out using the following steps: (1) the $200 \mathrm{~nm}$ line/space pattern was scanned 256 times at the scan pitch values shown in Table 1 (see Figure 2(a)); (2) the edge positions for each scan were determined (see Figure 2(b) and (c)); and (3) the 3-sigma and Power Spectral Density (PSD) for each scan pitch were calculated.

\begin{tabular}{|c|c|c|c|c|c|}
\hline Scan pitch $(\mathrm{nm})$ & 2.0 & 3.9 & 9.8 & 19.5 & 48.8 \\
\hline Total length $(\mu \mathrm{m})$ & 0.5 & 1.0 & 2.5 & 5.0 & 12.5 \\
\hline
\end{tabular}

Table 1. AFM measurement conditions.

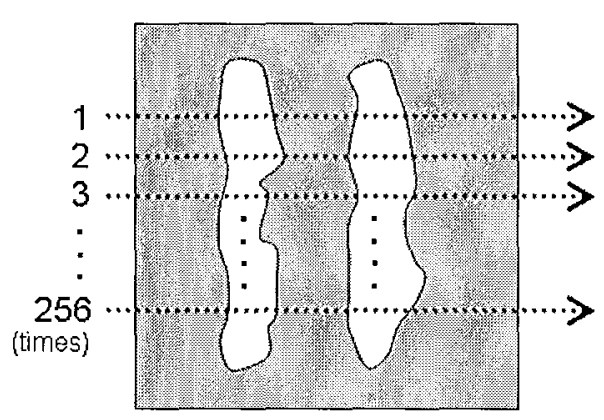

(a)

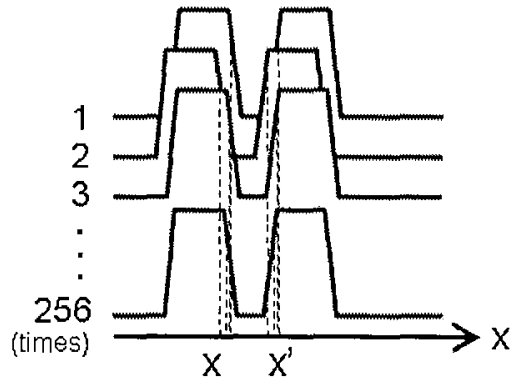

(b)

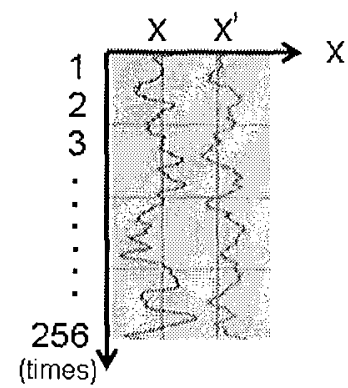

(c)

Figure 2. Procedure for mask AFM measurement. (a) Schematic of top-down view of line (white) and space (gray). Dashed lines shows direction of AFM scanning. (b) Schematic of AFM measurement data; $x$ and $x$ ' are the edges of lines. (c) Line edge profile from the 256 measurements of the locations of $x$ and $x$ '. 
For the mask SEM evaluation, measurement was carried out using the following steps: (1) Capture 200nm line/space images for every pattern density. (2)Load the SEM images to SuMMIT software and calculate 3-sigma of LER and PSD.

\subsection{Resist image evaluation}

The NOVACD EUV mask was printed in our current highest resolution resist using the ASML AIpha Demo Tool (ADT) in Albany, NY. This exposure tool has a central wavelength of $13.5 \mathrm{~nm}$, a $4 X$-reduction imaging system with a numerical aperture (NA) of 0.25 , and an illumination system providing a pupil fill of $\sigma=0.5$. Resist SEM images were recorded at the best focus and best dose for every pattern density. Then the images were analyzed using SuMMIT software.

\section{SURFACE ANALYSIS}

\subsection{Topography comparison by AFM and AIT}

The mirror and absorber surface of an unpatterned $400 \mu \mathrm{m}$ square area of the mask was scanned with an AFM using $100 \mathrm{~nm}$ square area/ $1 \mathrm{~nm}$ pitch.

In order to observe the surface with EUV light, the images of the $200 \mathrm{~nm}$ line/ space areas at various pattern densities were captured using the Actinic Inspection Tool (AIT) in Berkeley, CA. The AIT is an EUV microscope that uses EUV light from the Advanced Light Source synchrotron storage ring.

\subsection{Reflectivity analysis}

Reflectivity measurements were made using the AIT. Images of $30 \mu \mathrm{m}$ square mirror and absorber areas were recorded with various exposure time from 15 to 90 seconds. The reflected light intensity was calculated from the images to get the relative reflectivity between mirror and absorber.

\section{THOUROUGH CHARACTERIZATION OF 32NM TECHNOLOGY NODE SRAM}

\section{$4.132 \mathrm{~nm}$ technology node SRAM (M1) analysis}

A 32nm technology node SRAM (M1: first metal layer) pattern which was not corrected for optical proximity effects or for any EUV specific effects, was investigated from 5 viewpoints: mask SEM image, mask AIT image, near field image from simulation, wafer plane image from simulation, and EUV resist image printed with the ADT.

Simulation of the near-field and wafer-plane image of the SRAM was carried out using a finite-difference time-domain (FDTD) simulator called TEMPEST. The conditions used for the simulation are listed in Table 2 and the results of the simulation are discussed in Section 5.

Table 2 Simulation conditions of $32 \mathrm{~nm}$ technology node SRAM

\begin{tabular}{|c|c|c|}
\hline \multicolumn{2}{|c|}{ Incident wavelength } & $13.4 \mathrm{~nm}$ \\
\hline \multicolumn{2}{|c|}{ Numerical aperture (N.A.) } & 0.25 \\
\hline \multicolumn{2}{|c|}{ Angle of off-axis incidence } & 6 degree \\
\hline \multicolumn{2}{|c|}{ Illumination } & Conventional \\
\hline \multicolumn{2}{|c|}{ Effective source size } & 0.5 \\
\hline \multirow{5}{*}{ Mask sack } & Top $\mathrm{SiO}_{2}$ & $12 \mathrm{~nm}$ \\
\hline & TaN thickness & $55 \mathrm{~nm}$ \\
\hline & Bottom $\mathrm{SiO}_{2}$ & $10 \mathrm{~nm}$ \\
\hline & MoSi mirror & 40 pairs \\
\hline & Substrate & --- \\
\hline \multicolumn{2}{|c|}{ Magnification } & $4 x$ \\
\hline
\end{tabular}




\section{RESULT AND DISCUSSION}

\subsection{LER 3-sigma evaluation by mask AFM and mask SEM}

Figure 2 shows examples of the line edge profiles that were collected by AFM measurement. In the case of $90 \%$ pattern density and $500 \mathrm{~nm}$ measurement length, the line edge profile shows a wavy form. However, as measurement length increases, the wavy form is increasingly dominated by noise. As pattern density decreases, the wave height becomes lower and lower.

We use these line edge profiles to calculate 3-sigma and PSD. These data will be presented after the mask SEM results.

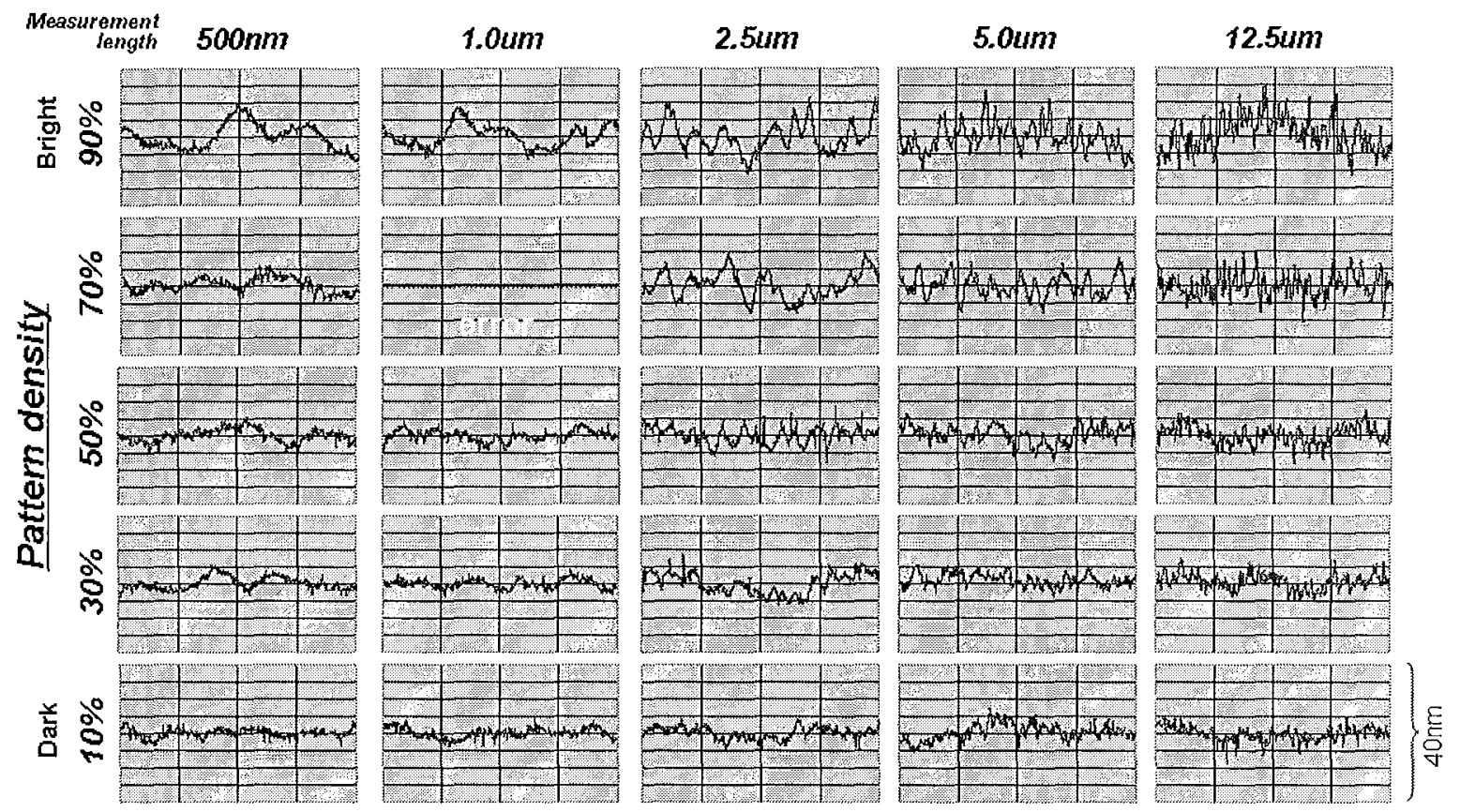

Figure 2. Examples of line edge profile by AFM. Full range of $x$ axis in left most column is $500 \mathrm{~nm}$. The $2^{\text {nd }}, 3^{\text {rd }}, 4^{\text {th }}$, and $5^{\text {th }}$ columns are $1.0,2.5,5.0,12.5 \mu \mathrm{m}$, respectively. Top row graphs are the $90 \%$ pattern density case. $2^{\text {nd }}, 3^{\text {rd }}, 4^{\text {th }}$, and $5^{\text {th }}$ rows are data for 70,50 , $30,10 \%$ pattern density, respectively. Full range of $y$ axis for all graphs is $40 \mathrm{~nm}$.

Figure 3 shows SEM images of the NOVACD mask. LER is quite low from $1 \%$ to $30 \%$ pattern density. However, as the density increases above $40 \%$, the LER becomes rougher and rougher. 


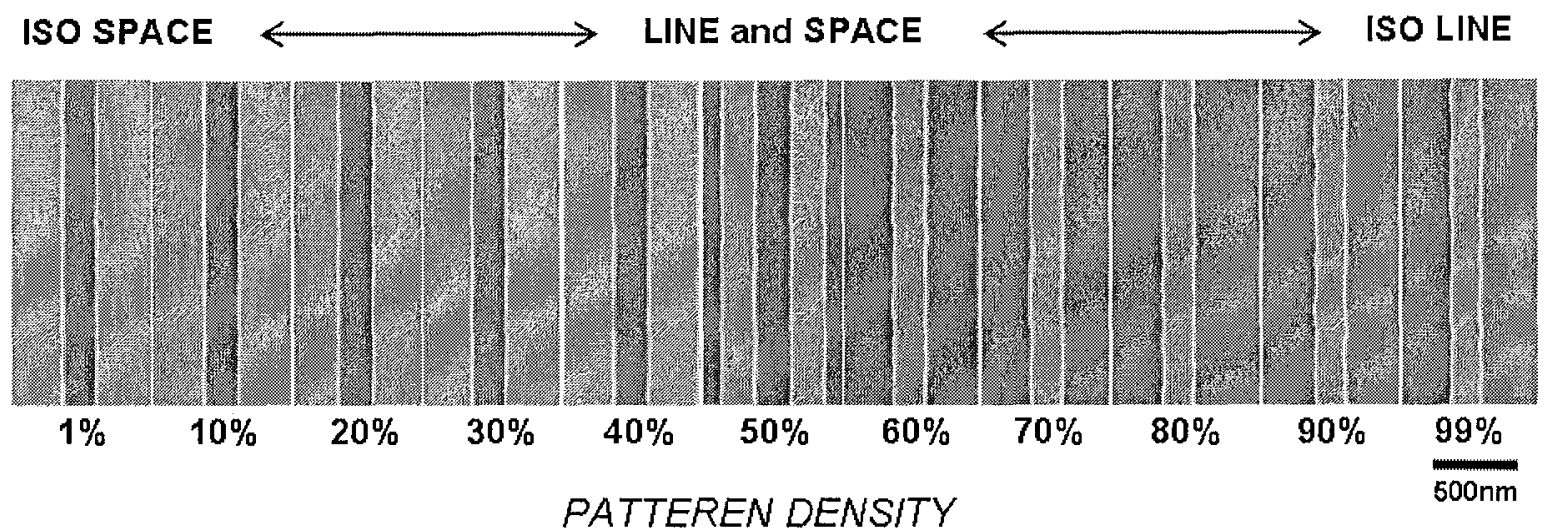

Figure 3. 200nm line and space image of mask. Black and gray images are mirror and absorber area, respectively. From $1 \%$ to $50 \%$, center pattern is mirror area. From $60 \%$ to $99 \%$, center pattern is absorber area.

Figure 4 shows LER (3-sigma) values from both AFM and SEM data. The SEM data shows good correlation to the AFM data. If we use an average of the AFM data, there is about $1.5 \mathrm{~nm}$ offset to the SEM data. If we use the $500 \mathrm{~nm} \sim 1.0 \mu \mathrm{m}$ scan length range, SEM data is in good agreement with the AFM data. This means that SEM measurements can be used to accurately evaluate mask LER. However, as the AFM scan length increases at each pattern density, the LER goes up. This means that an SEM may not be able to accurately measure long range LER. Because of this, we have compared AFM PSD data with SEM PSD data.

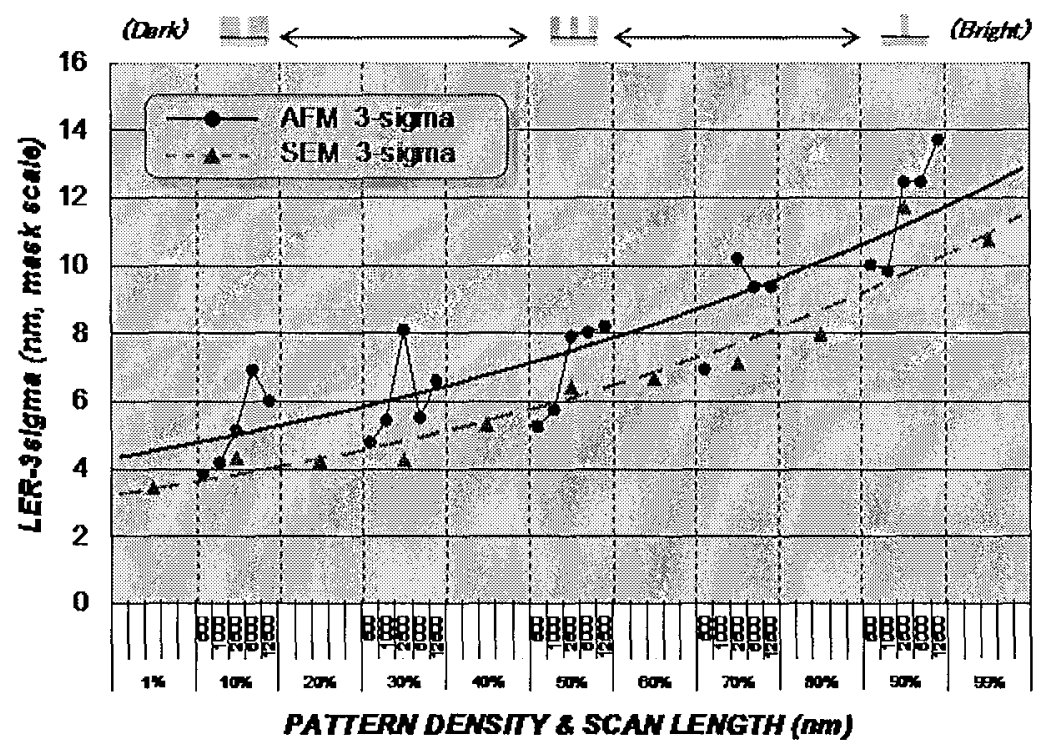

Figure 4. Pattern density and scan length dependence of LER (3-sigma). The circles and the solid line show mask AFM data. The line shows the best fit for the average of 5 points at each pattern density. The triangles and the dashed line show mask SEM data and the best fit for the plots, respectively.

Figure 5 shows a PSD comparison of AFM and SEM data. As pattern density decreases, the AFM measurements show that from (a) $90 \%$ to (e) $10 \%$, the slope of the PSD becomes more gradual. However, the SEM result shows that all 
pattern densities show almost the same slope. This means that the AFM data is more sensitive to periodic roughness than the SEM data. The PSD plots clearly show that mask LER is comprised of a broad spectrum of spatial frequencies.

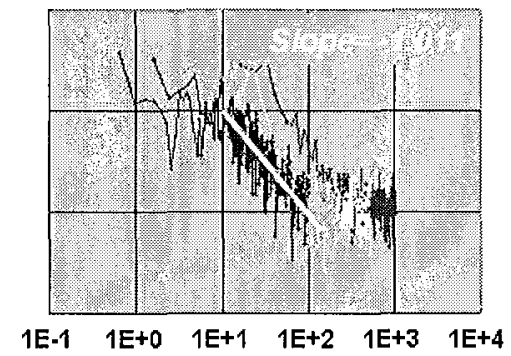

SPATIAL FREQUENCY (1//, $\mathrm{m}$, wafer scale)

(a)

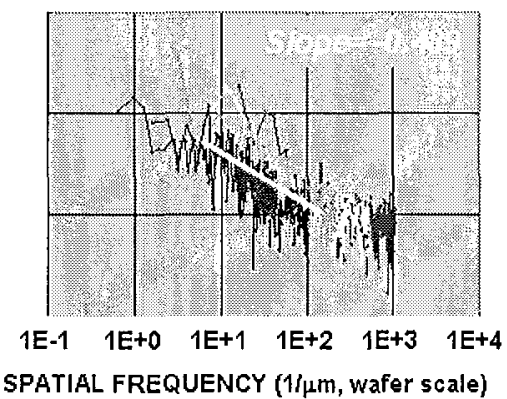

(d)

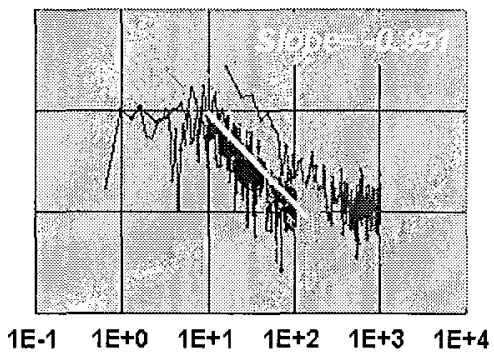

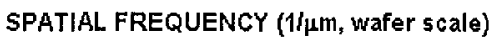

(b)

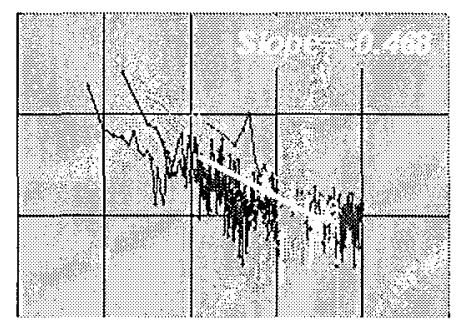

$\begin{array}{llllll}1 E-1 & 1 E+0 & 1 E+1 & 1 E+2 & 1 E+3 & 1 E+4\end{array}$ SPATIAL FREQUENCY (1//1m, wafer scale)

(e)

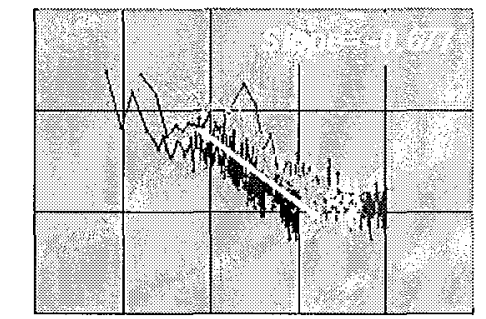

1E-1 1E+0 1E+1 $1 E+2 \quad 1 E+3 \quad 1 E+4$

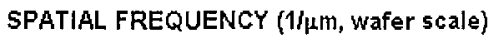

(c)

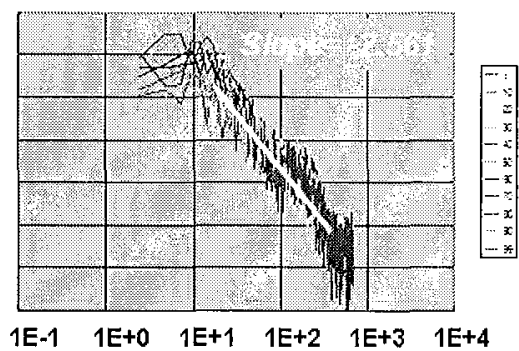

SPATIAL FREQUENCY (1/1/m, wafer scale)

(f)

Figure 5. PSD of mask LER from the AFM data. The graphs (a) to (e) are PSDs for the $90,70,50,30$ and $10 \%$ pattern density cases, respectively. Each graph includes all of the data at all measurement lengths shown in Figure 2. The graph (f) is PSD of mask LER for the SEM data at all pattern densities.

\subsection{LER 3-sigma evaluation of resist printed image}

Figure 6 shows examples of resist images of 50nm line and space. Figure 7 and 8 are resist LER and PSD from mask AFM and mask SEM data.

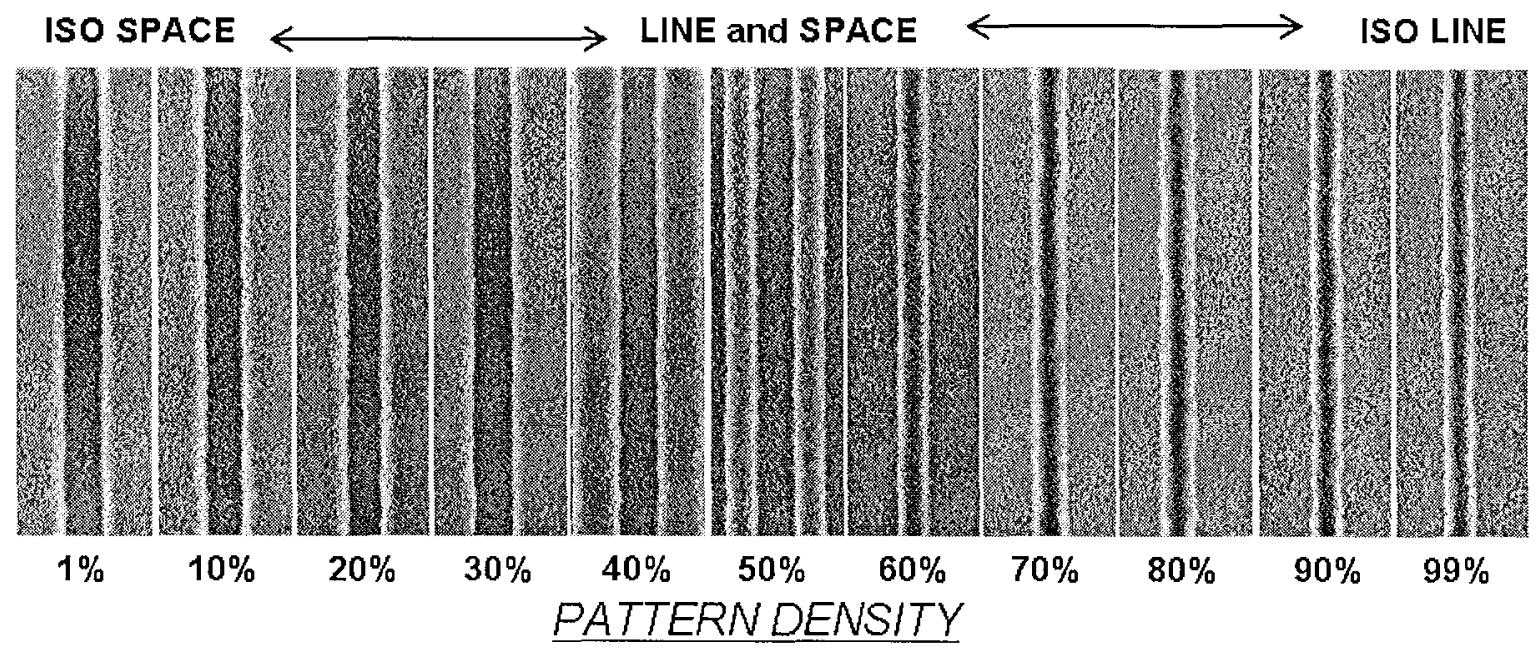

Figure 6. Resist SEM image of 50nm line and space patterns. 
In Figure 6, as pattern density increases, the resist LER increases gradually. In Figure 7, resist LER remains about 2.3nm over the 1 to $40 \%$ pattern density range. However, above $50 \%$ pattern density, the LER gets larger and larger. It seems that there is no correlation between resist LER and mask SEM /AFM LER. Possible reasons for this are resist blur, image log slope, SEM image quality, and so on.

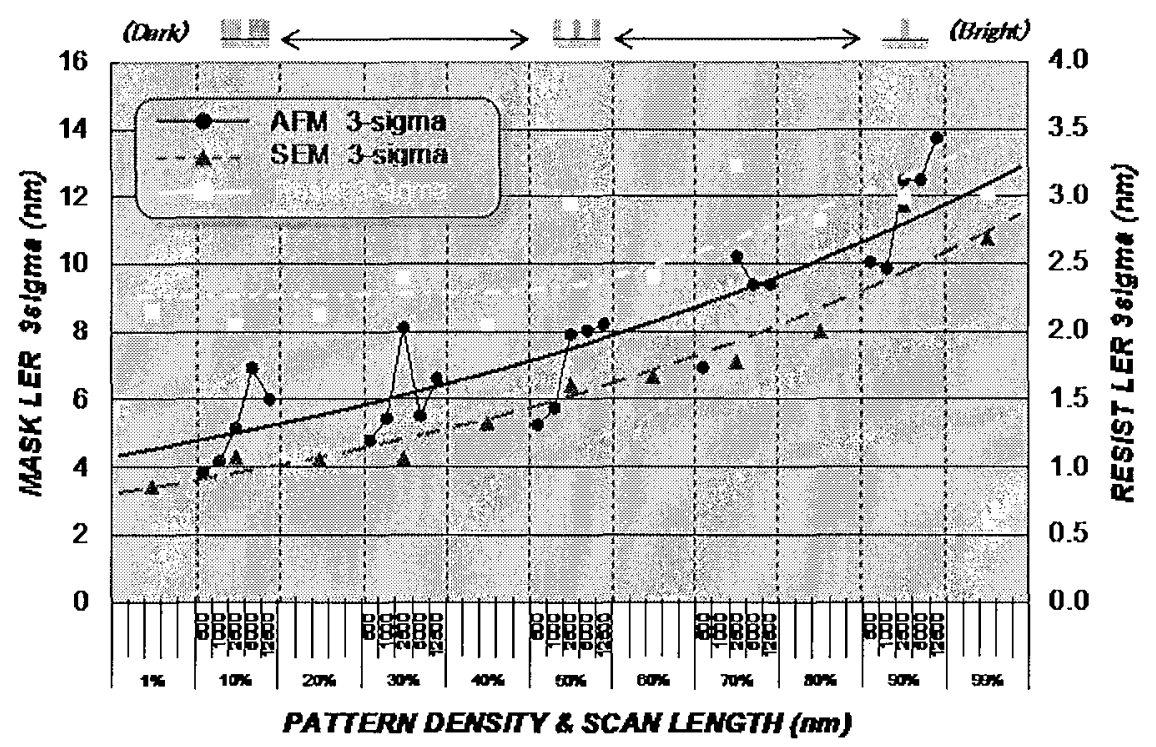

Figure 7 Pattern density dependence of resist LER and mask LER. The mask LER data is the same as that shown in Figure 4.

Figure 8 shows LER PSD comparison of the mask AFM, mask SEM and resist SEM data. All pattern densities of resist LER fall on one line that has the same trend as the mask SEM data. These data also show that the AFM data may be more sensitive to periodic roughness than the SEM data, even if it involves a resist measurement.
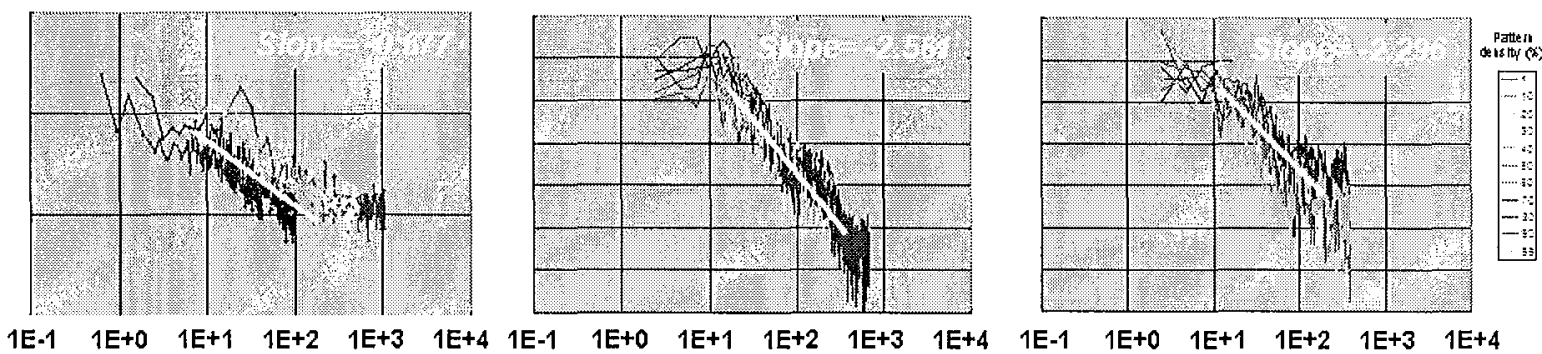

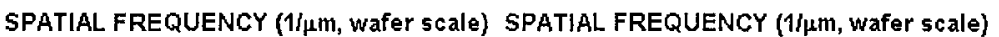

(a)

(b)

(c)

Figure 8 LER PSD of (a)mask at 50\% pattern density by AFM measurement, (b)mask at all pattern densities by SEM measurement and (c)resist at all pattern densities by SEM measurement. (a) and (b) are same graphs shown in Figure 5 (c) and (f). 


\subsection{Surface roughness analysis}

Figure 9 shows the topography of the mirror and absorber surface recorded with an AFM. The mirror and absorber roughness numbers are about 6 and 7 angstrom rms, respectively. In order to better understand the effect of this roughness, EUV images in each of these areas were recorded using the AIT.

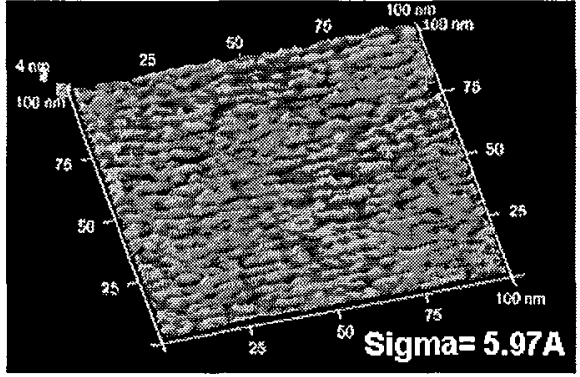

(a)

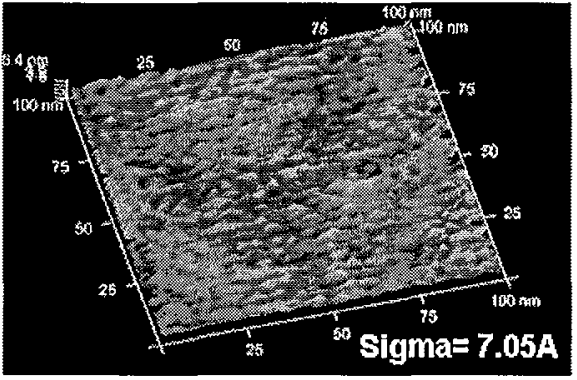

(b)

Figure 9. Surface roughness measurement of mirror and absorber using an AFM. Scanned area was 100nm square. (a) and (b) show mirror and absorber areas, respectively and the value for rms roughness is included in each image.

Figure 10 shows AIT images of $200 \mathrm{~nm}$ line/ space at various pattern density. AIT shows good contrast at all pattern densities. Figure 11 is a magnified view of the line shown in Figure 10 (99\% pattern density). Since there is some roughness in this image, this indicates that EUV light interacts with the mirror roughness. It is possible that phase roughness is cause of this, even though the wavefront error in the AIT is small. ${ }^{9}$

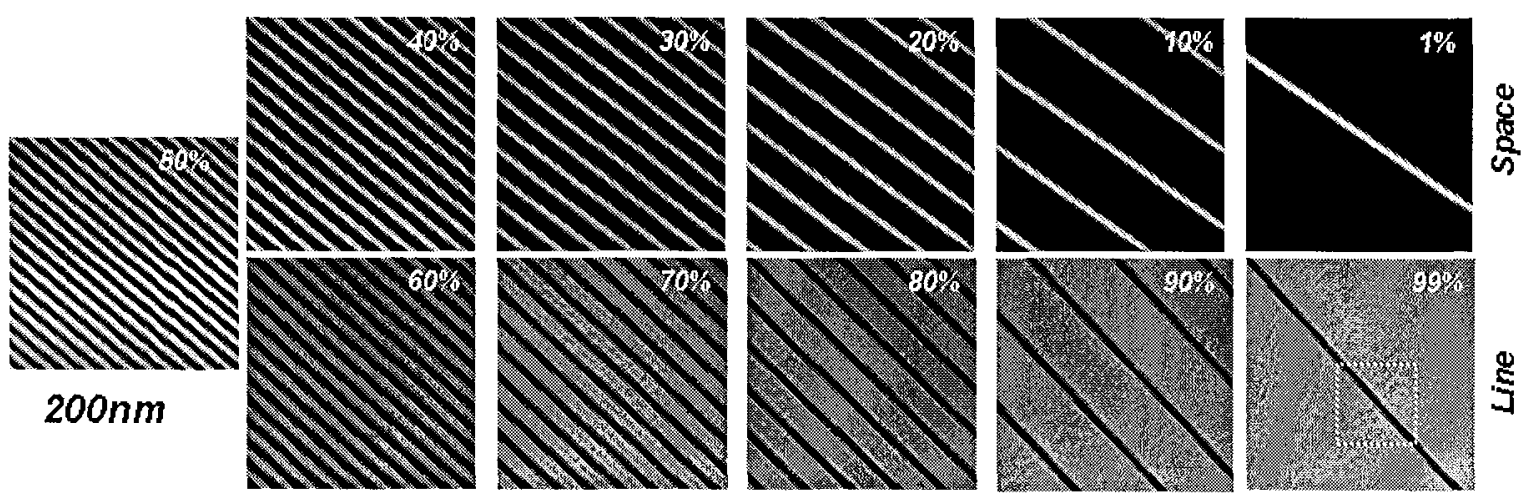

Figure 10. AIT images of 200nm line and space at various pattern densities. 


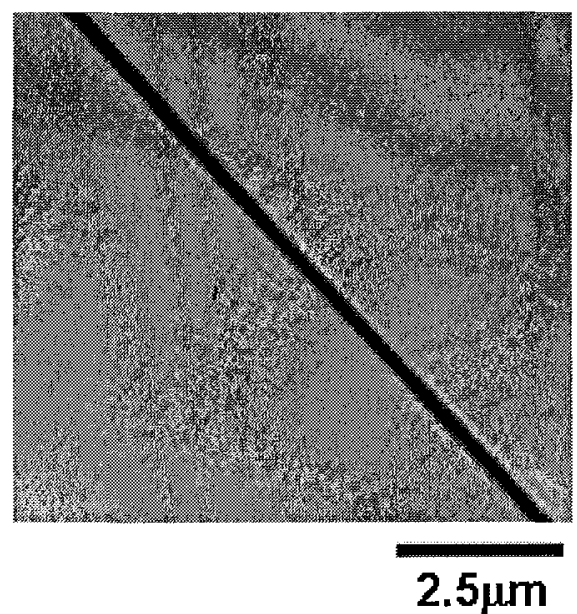

Figure 11. Zoom up image of AIT from Figure 10.

\subsection{Reflectivity analysis}

Figure 12 shows the reflectivity measurement results. The images show clear intensity changes with exposure time. The intensity of both mirror and absorber show good linearity with exposure time. The ratio of the two slopes shows that mirror reflectivity is 19.9 times higher than absorber reflectivity. If we assume that the range of mirror reflectivity is $60 \sim$ $68 \%$, then the absorber reflectivity is $3.00 \sim 3.42 \%$.
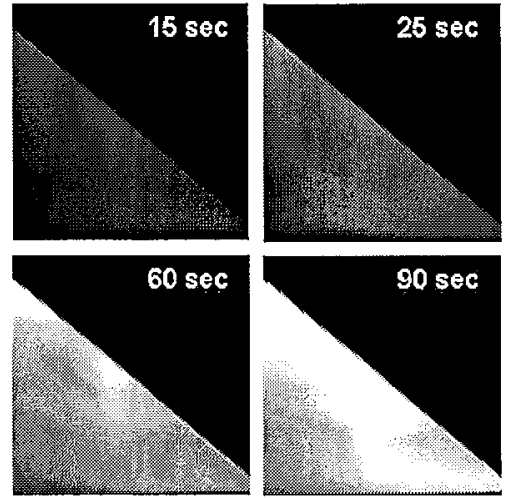

(a)

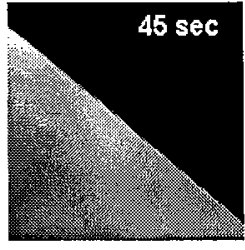

$\overline{10 \mu \mathrm{m}}$

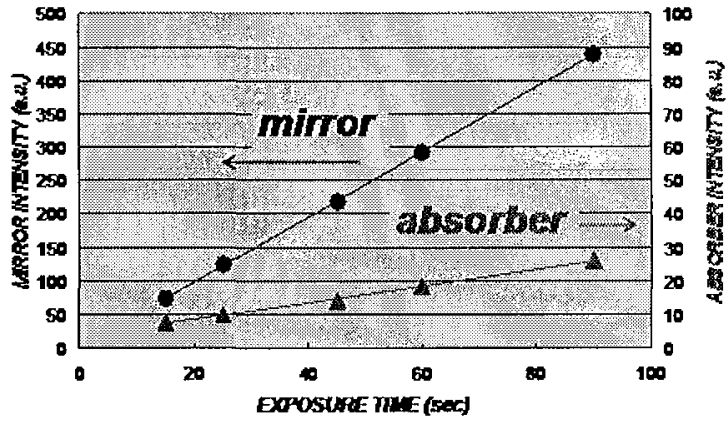

(b)

Figure 12. Mask reflectivity measurements. (a) shows AIT images of mirror (bottom left) and absorber (top right) at various exposure times. The exposure times are shown in each image. (b) shows reflection light intensity of mirror and absorber. 


\section{$5.532 \mathrm{~nm}$ technology node SRAM (M1) thorough characterization}

Figure 13 shows the results of a thorough characterization of the SRAM area on the mask. The mask SEM image (a) shows contrast and no defects. The mask image corresponds to overlaid image of (b) quite closely. These results show that the mask fabrication process was good. The near field image of the scanned area captured with the AIT (c) shows bright and dark areas. This may be due to non uniform illumination of the mask. At this time, the image quality of the magnified image (d) is not as good as current AIT image quality. ${ }^{10}$ However the contrast is still quite nice.

Near field image simulation results of (e) and (f) also show very good contrast. After calculating the wafer plane image, we apply Gaussian blur values of 0,10 , and $20 \mathrm{~nm}$ in $(\mathrm{g})$. As the blur increases, image contrast decreases. However, the contrast is obviously good enough to print, because the patterns are not merged and none are missing.

Even though the SRAM patterns were not corrected, the resist SEM image (h) shows no defects, no missing patterns, and no merged patterns. It is hard to see the difference between the printed image and simulation result in (i). This means that the ADT imaging of the $32 \mathrm{~nm}$ technology node SRAM is quite good and is predictable by simulation.

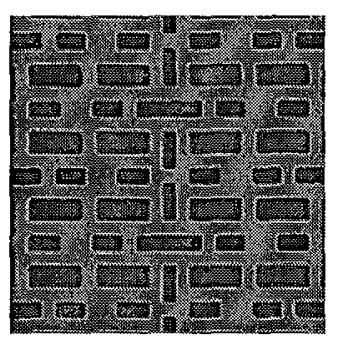

(a)

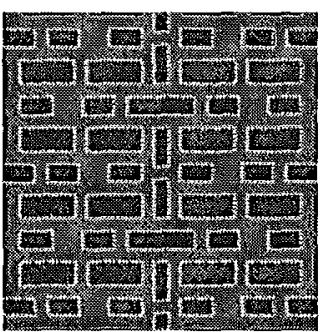

(b)

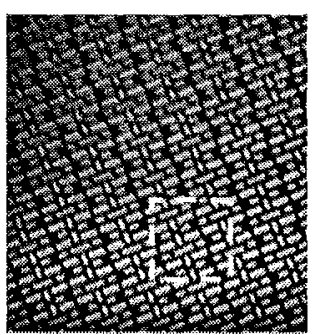

(c)

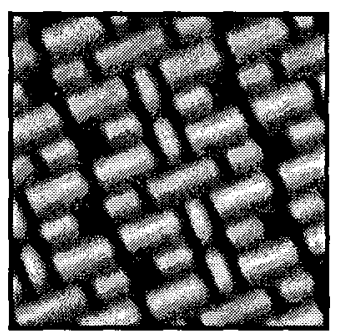

(d)

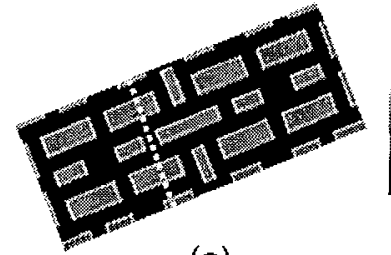

(e)

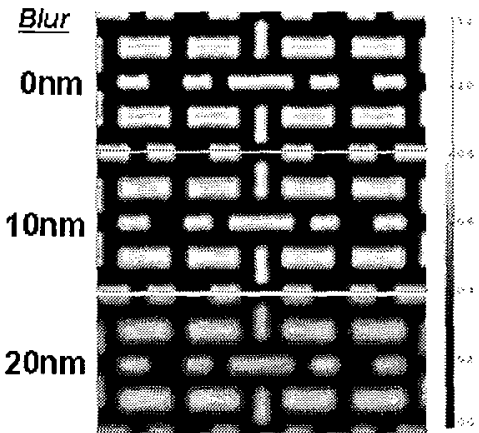

(g)

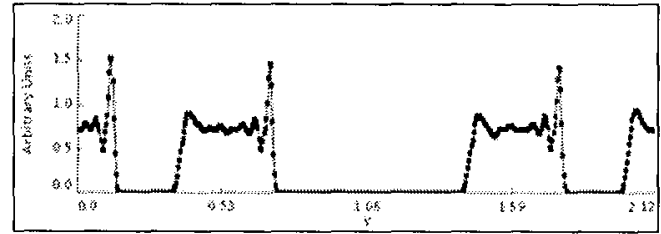

(f)

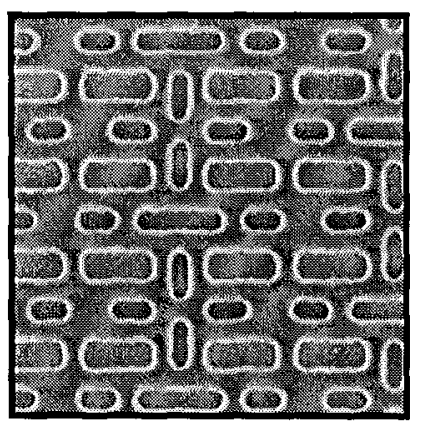

(h)

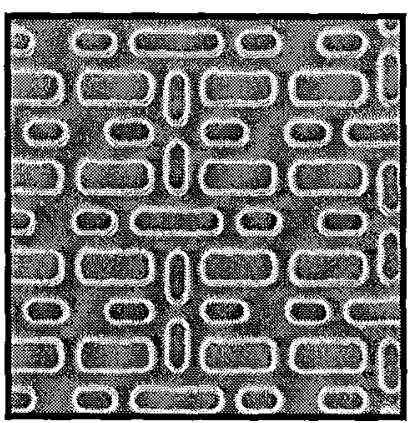

(i)

Figure 13 Thorough characterization of $32 \mathrm{~nm}$ technology node SRAM (M1) pattern. (a)Mask SEM image. (b)Mask GDS data overlaid on the mask SEM image. (c)Mask AIT image. Incident light comes from the bottom of the page with 6 degree off axis. (d)Magnified image of dashed rectangular area. (e)Simulation result of near field image. Incident light condition is same as (c). (f)Cross section of near field image intensity of white line in (e). (g)Simulation result of wafer plane image intensity with 0,10 and $20 \mathrm{~nm}$ blur values. (h)Resist image printed with the ADT. (i)Wafer plane image simulation result with 10nm blur (white line) overlaid on the image in $(\mathrm{h})$. 


\section{SUMMARY}

In order to study EUV mask effects a thorough characterization of one mask, a so-called NOVACD mask, was carried out. LER determined from mask AFM and mask SEM were found to be well correlation for $200 \mathrm{~nm}$ line and space at various pattern densities when the length of AFM scanning was $500 \sim 1000 \mathrm{~nm}$. The contribution of mask LER was found to be much smaller than resist imaging LER.

AFM scanning and AIT imaging were used to analyze mask surface roughness and to measure the reflectivity ratio between mirror and absorber was measured. The AFM data show 6 and 7 angstrom rms for mirror and absorber surface, respectively. The intensity contrast in the bright areas of the AIT images is related to reflected phase roughness inherent in the mask, coupled with the aberrations of the AIT.

Thorough characterization of a $32 \mathrm{~nm}$ technology mask SRAM M1 pattern was carried out and a mask SEM image, an AIT image, a resist printed image, and simulation were shown. The ADT images were of high quality even though the mask patters were not corrected for OPC or any EUV-specific effects. Image simulation results are in good agreement with the resist printing results.

\section{REFERENCE}

[1] B. LaFontaine, et al., "The use of EUV lithography to produce demonstration devices", Proc. SPIE. Vol. 6921, 69210P (2008)

[2] O. Wood, et al, "Integration of EUV lithography in the fabrication of 22-nm node devices", Proc. SPIE (2009) (in printing)

[3] H. Meiling, et al., "Performance of the Full Field EUV Systems", Proc. SPIE, Vol. 6921, 69210L (2009).

[4] H. Aoyama, Y. Tanaka, T. Kamo, N. Iriki, Y. Arisawa and T. Tanaka, "Flare evaluation for 32-nm half pitch using SFET", Proc. SPIE, Vol. 6921, 69213H (2008).

[5] H. Mizuno, et al., "Flare evaluation of an ASML Alpha Demo Tool", EUVL Symposium (2008)

[6] H. Mizuno, et al., "Flare evaluation of ASML Alpha Demo Tool", Proc. SPIE (2009) (in printing)

[7] M. Sugawara, "Assessment of pattern position shift for defocusing in EUV lithography", Proc. SPIE. Vol. 6517, $65170 \times(2007)$

[8] G. McIntyre, et al., "Modeling and Experiments of Non-Telecentric Thick Mask Effects for EUV Lithography", Proc. SPIE (2009) (in printing)

[9] P. Naulleau, K. Goldberg, I. Mochi, G. Zhang, "Mask Effects on LER,"EUVL Symposium (2008)

${ }^{[10]}$ I. Mochi, et al,. "Improving the performance of the Actinic Inspection Tool with an optimized alignment procedure", SPIE (2009)

\section{ACKNOWLEDGEMENT}

This work was performed by the Research Alliance Teams at various IBM Research and Development Facilities

The authors would like to thank Masamitsu Ito, Takashi Hirano and Rikiya Taniguchi of Toshiba Corporation and Takumichi Sutani of Hitachi America.

This work was supported by the Director, Office of Science, of the U.S. Department of Energy under Contract No. DE-AC02-05CH11231. 